\title{
Endodontic Failures-A Review
}

\author{
${ }^{1}$ Dr. Sadashiv Daokar, ${ }^{2}$ Dr.Anita.Kalekar \\ ${ }^{1}$ Professor and P.G. Guide Department of Conservative Dentistry, C.S.M.S.S Dental College, Aurangabad . \\ ${ }^{2}$ Post-graduate student Department of Conservative Dentistry C.S.M.S.S Dental College, Aurangabad.
}

\section{Introduction.}

The term success or failures in endodontics must be defined rigidity, in order to be meaningful. A clear definition \& agreement of what constitute a failure following endodontic treatment does not exist among endodontist.

The dentist had reduced criteria for success of endodontic treatment to a very narrow definition to absence of pain. How convenient it would be if this concept could be totally accepted. Unfortunately absence of pain is not completely a reliable measure for good health or success in endodontic treatment.

Countless peoples are living today with some disease in its pain free stage after endodontic treatment. Endodontic treatment apparently success's in some cases in spite of, our best efforts. These fortunate circumstances can also be attributed to a tremendous capacity of body's natural defences to cope with infections and to enhance the body's survival rate.

Practicing endodontist should know that lack of pain is not sole criteria of success of endodontic treatment, but they would be hard pressed to present universally acceptable criteria for success or failures.

According to seltzer samul (1988) the use of term adequate clinical function is more realistic, because the retention of tooth in function is ultimate goal of endodontic therapy.

Failures may also be based solely upon clinical conditions, i.e. the persistence of development of symptoms such as discomfort, pain, swelling or sinus tract, despite of negative radiographic finding.

\section{Radiographic Criteria.}

Among some endodontists, more rigid criterias are employed. They stipulated that the criteria for failure are 1) Development of radiographic rarefaction of periapical area after completion endodontic treatment.2) in cases, where none has been present before treatment appearance of radiographic rarefaction of periapical area after endodontic treatment. 3) The increase in size of area of rarefaction after completion of root canal treatment. (Bender et al-1964,Luebike et al -1964,Seltzer et al -1963)

Radiographic evaluation plays basic role in the assessment of treatment result any falliability associated with the interpretation of radiograph directly distort the reported rate of success and failure.

\section{Histologic Criteria.}

Following operative procedure, pulp inflammation may persist for many year frequently, judged by the absence of pain or positive radiographic finding neither operator nor patient aware of this phenomenon.

In other words procedure has been successful clinically, yet histologically pulp lesion may present $\underline{\text { seltzer et al }}$ $-1963)$.

It should not assume that following endodontic treatment periapical lesion always resolves.

\section{Timing Of Endodontic Failure.}

Failure in endodontically treated teeth occur at various time interval up to 10 year or more after completion of treatment(seltzer et al -1967). Our studies have revealed that the majority of the unsuccessfully revealed teeth failed within 24 months of completion of treatment other failed up to 10 years \& a few failed more than 10 years.

Thus, for assessment of result a 2 year period is better than a 6 months follow-up period in teeth without area of rarefaction, but a 6 month check up period is adequate for evaluation of trends in teeth with area of rarefaction.

\section{Root Canal Therapy Is Called Successful If.}

1) The treated tooth is asymptomatic \& functional.

2) Soft tissue appears normal \& responds normally to manual examination.

3) Radiograph reveals a normal lamina Dura. 


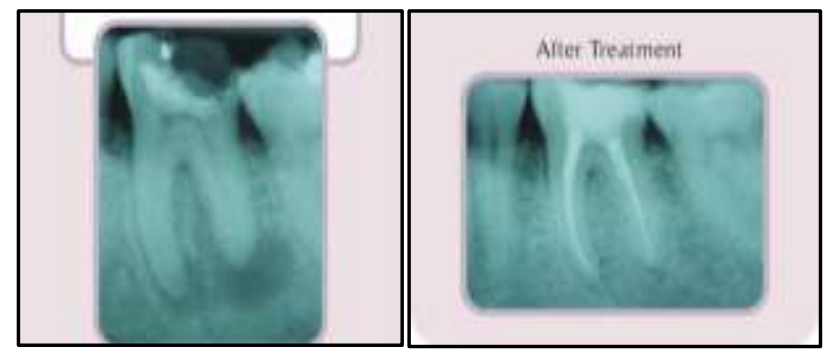

TREATMENT IS CONSIDERED FAILED IF.

1) Treated tooth is symptomatic or has an abnormal appearance.

2) Soft tissue response abnormally to manual examination.

\section{RADIOGRAPHIC CHANGES ALSO REVEAL THE FAILURE.}

1) When a lesion remains the same radiographically or become diminished in size only, after the treatment, it indicate incomplete repair.

2) When periapical lesion appears subsequent to endodontic treatment. or if a pre-existing lesion increases in size.

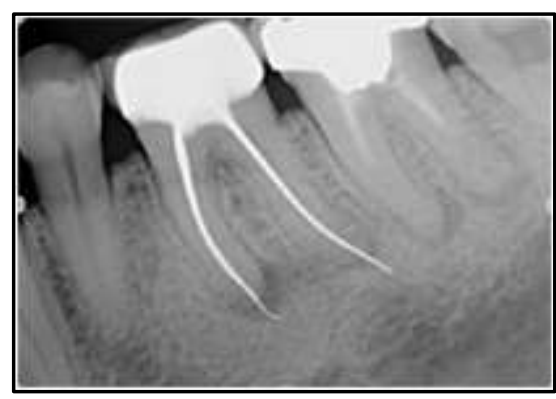

\section{A. Preoperative causes.}

\section{Causes Of Endodontic Failures.}

\section{1) Incorrect diagnosis.}

- It can be result of misdiagnosis, poor case selection, \& poor prognosis.

- Incorrect diagnosis usually results from a misinterpretation or lack of information, either clinical or radiographic.

- Errors in case selection are not so easily overcome as operative error \& may after be listed as the bad brakes of game rather than error in judgement.

- A careful medical history is tune for essential.

2) Endo- periodontal lesions.

- Of all the cause of endodontic failure, that is determined prior to treatment, the concomitants periodontal periapical lesions may be discovered more frequently.

3) Altered canal space.

- Calcification

- Due to inflammatory response to caries calcification occur in root canal it is decalcified by chemical material EDTA \& subsequent root retreatment successful.

4) Traumatic injuries.

- It has beer commonly observed that, teeth requiring endodontic treatment after traumatic injury have a higher rate than do teeth with pulpal problem of the others origin .

- Root resorption caused due to the traumatic to the attached apparatus result in failure.

5) Internal resorption.

- In such teeth over extension of the filling must be avoided by creating stop in the dentin at short of radiograph apex. 
6) Systemic diseases.

- Several systemic diseases interfere with the regenerative capacity of bone. These include Paget's disease, hyper parathyrodism, hyperthyroidism, and osteoporosis.

- Other factors like age, nutrition, hormones, chronic disease, vitamins, stress and dehydration also affect the periapical repair.

- The systemic disease may influence local tissue resistance, there by interfering with healing potential. In addition, systemic disease may frequently ductile modification of treatment procedure.

\section{B. Operative causes.}

1) Anatomical Variations.

- According to Ostrander (1958) such factors as presence of excessively curved canal, excessive root mineralization, impenetrable accessory canal \& canal bifurcation near root open may result in endodontic treatment failures.

\section{2) Technical difficulties.}

- Operative procedures in endodontic therapy are aims at to obtaining mechanical \& biologic objective, assuring through cleaning \& reshaping of root canal system. Negligence to observe those objectives may result in treatment failure.

- $76 \%$ failure occurs.

3) Infections.

- The presence of infected \& necrotic pulp tissue within root canal act as a continuous irritant to the periapical tissue \&there necessitate through debridement of root canal

- Ostrander E C (1958) claimed that some endodontic cases probably fail because strictly aseptic technique was not followed \& new microorganism introduced into the root canal during treatment.

\section{4) Poor debridement.}

- Untreated or inadequate debridement of root canals has a definite relationship to the failure of endodontic treatment (Grossman 1972).

- Debridement of root canal reduces the microbial flora, but apparently does not eliminate it (Ingle 1965).

\section{5) Broken instruments .}

- $\quad$ The presence of denticles in the root canal complicates endodontic therapy. When situated in apical third of root canal, denticles enhances the possibility of breakage of barbed broach especially there is defect in steel. Consequently barbed broach should neither be introduced into tight canal, not they should be used for enlargement of canal but merely to engage the pulp tissue for removal. Commercially available retrieval kits can also be used for broken instrument removal.

6) Mid-treatment flare up.

- The prompt \& effective treatment of mid treatment flare ups of all type is essential \& integral part of endodontic therapy.

- The expeditors' management of these flare -ups enhances a positive attitude amongst patient towards endodontic treatment. Otherwise, patient may lose interest in continuing the treatment which ultimately returns in failur.

7) Mechanical \&chemical irritants.

- When instrument has been confine to root canal \& presumably instrument root have damage the apical pulp stump, chances for repair are enhanced (Strindberg 1956).

- Strindberg (1956) found that, even teeth with non-vital pulp. There was lower frequency of failure when canal could not be reamed through apex as compared to these where instrumentation was carried out to or beyond the apex.

- Grahnen \&Hansson (1961) \&Frosteu (1963) even found that failure frequency was greatest in single noted teeth when the canal is apparently easiest to file \& ream.

- During endodontic treatment, various medications are used as dressing in root canal. Their functions are presumably to eliminate or reduce microbial flora, prevent or lessen pain, reduce inflammation or stimulate repair.

- Torreck (1961), Schilder and Amesterdam (1956) have demonstrated irritating potential of many root canal medicament. 
- Successful endodontic therapy does not require use of drug (Matsumiya \& Kitamar 1960).

8) Access preparation.

- An access opening which is misaligned \& under extended often causes root of furcai perforations \& tooth loss unless surgically corrected.

- Small or incorrectly placed access cavities\& the openings misaligned from the long axis of the tooth results in failure, debris \& the bacteria in the pulp horns must be allowed to remain.

9) Excessive haemorrhage.

- Small haemorrhages during endodontic procedure are repaired without incident.

- But, excessive haemorrhage when occur due to extirpation of an inflamed pulp together with instrumentation beyond the apex of the tooth, it result in mild inflammation, due to formation of haematoma by local accumulation of blood. In these cases, the extravagated blood cells and fluid. If not resorbed by macrophages, repair is delayed in the presence of infection of the extravasiate blood act as a nidus for bacterial growth (Seltzer et al 1988).

10) Under extended filling.

- Under extension can be seen on radiograph and in such cases retreatment is the first consideration solvent like zylene can be used to remove gutta-percha, but care must be taken that such product should not force into the periapical area during gutta-percha removal procedure.

- If required surgical approve become necessary to correct the underfillings.

\section{1) Over extended filling.}

- Over extension of root canal filling, gutta - percha, sliver point delays periapical heating.

- Selzar et-al (1972) performed SEM of the sliver cone studies revealed that the corrosion of the sliver cone was associated with sulphur. Chemical product formed predominantly a sliver amine sulfatelamide, all cells body contain sulphur primarily.

- Numerous studies have shown that highest success rates were obtained when root canal terminates 1-3 mm from the radiograph open (Strindberg 1956)

- Radiographically, the overfill can usually be discerned as the filling material is seem to expand, curt, curve or lacks in the symmetry.

- According to Crump (1979), it is not necessary to treat overfill unless clinical symptoms develop can be operated to obtain the radiograph pleasing result.

- Overfilling showed is avoided for the successful of the root canal therapy.

- Causes of overfilling:

1. Failure in determine the exact location of the apical foramen an absence of apical stop or constriction in mature teeth.

2. Incorrect selecting of master cone.

3. open apices.

12) Sliver points corrosion.

- Silver sulphides, silver sulphate, silver carbonates \& silver amine sulphate amide hydrate are the compound formed after corrosion.

13) Improper obturation

- Failure also occurs, when gutta-percha appears to fill the canal to the apical foramen, but does not obliterate the canal space laterally.

- If master cone binds tightly in the middle third or coronal third of the canal, but fails to the apical third.

- The tug-back resistance originates in the wrong area, because accessory cones fail to pass apical third of the canal after lateral condensation.

14) Improper placement of posts.

- Improper placement of posts \& use of the large posts also results in failure.

- Inadequate post extension leads to failure.

15) Corrosion of posts.

- It has been observed that, copper gets migrated towards the dentin attached soft tissue also contains silver \& iron. 
- According some investigators, such migration of corrosion product into dentin may result in root fracture.(Arhlidson k \&wroblewski r 1974).

16) Iatrogenic causes.

- Mechanical perforation is common cause of endodontic failure.

- Perforation generally occurs when the dentist is disoriented as to the direction of the bur \& its relationship to the anatomy of the pulp chamber or root.

- High potential area for furcation floor of molar \& two rooted maxillary pre molars roots that are narrow mesiodistally \& broad bucco-lingually with cured canals.

- Occur common frequently during high stress situation.

\section{a) Furcation perforation.}

- Frank has found the repair of furcation perforation is unpredictable because of the periodontal communication. He suggests considering sectioning of the tooth or root removal (Frank 1974).

- However seating material in the furcation perforation amalgam proved superior or cavit or calcium hydroxide applied immediately to such a lesion may have merit.

b) Mid-root \& apical third perforation.

- Should immediately sealed if possible or calcium hydroxide used prior to sealing.

- If the perforation is excessively large or long standing a full vertical flap should be reflected and the area repaired with zinc free amalgam.

- If the location of perforation is near the apex, an apiectomy is more handing the case.

D.Post-operative causes.

1) Failure following retreatment.

- Once endodontic treatment has failed, the chances for second failure after non surgical retreatment we enhanced (Bergenholtz et al 1979)

2) Failure following surgical retreatment.

- Failure of endodontic treatment may occur following apiectomy, apical curettage

- As per the block etc (1978) success rate after surgical retreatment is $67 \%$, 3\% failure outcome of $40 \%$ was uncertain.

Strindberg $L Z$ (1956):- endodontic failure is about six decade old story started by Strindberg by his comprehensive study and has made attempt to answer the basic questions about the success and failure of endodontic treatment.

Jokinen et al (1978), Morse et al (1983) \& Pekruhn (1986)- conducted study to assess the success and failure rate of endodontic treatment. Success rate ranged from 53\%-95\%.

Grossman et al(1965) :- conducted study of 1229 cases treated by student \& practitioner, success rate was $91.5 \%$ while failure was $8.5 \%$.Divided the causes of endodontic failure in four category i.e. poor diagnosis , poor prognosis , technical difficulties \& careless treatment(1972).

Torpe, Transtad \& Maltzdo: - Described the contribution of overextended access preparation leads to exc.ess reduction of dentin leads to weaking of tooth.

Seltzer s,Sinai \& Agust D(1970) :Irritating effect or the micro leakage of materials used for repair of perforations causes endodontic lesion to develop and subsequent failure of treatment.

Grossman LI (1972) : Objective of endodontic treatment is complete removal of potential irritant from root canal space, control of infections, shaping of the canal and removal of organic debris is considered as critical requirement of endodontic therapy to do successfully.

Crumps (1979):- Has listed several alternatives for differential diagnosis of endodontic failures i.e. perforation, obturation, overfilled, root canal missed, periodontal disease, another tooth and trauma.

Szajkiss and Fagger M (1983) :- said that more rigid the criteria of operator in case selection, greater the chances of success, where as treatment of all cases without case selection criteria increases the chances of failures.

Ingle (1985) :- Described causes of endodontic failures into three main groups. Apical perforation, operative errors and errors in case selection.

Kaffe I and Saint Louis (1987) :- Describes that incorrect diagnosis resulted from a misinterpretation or lack of information either clinical or radiographic leads to endodontic treatment in wong tooth and causes subsequent failures of endodontic treatment 


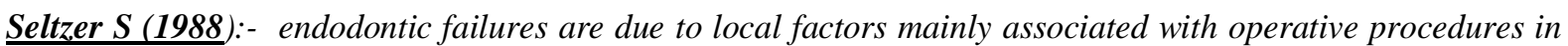
root canal therapy.

Maloney LG Feik SA and Ellender (1993) :- Showed microleakage of materials like EBA cement, amalgam, glass ionomer cement used for repair of lateral wall perforations during endodontic treatment which contributes in adverse results of endodontic treatment

Ray HA \& torpe M (1995) :- Described the relationship between endodontic treatment and restorative dentistry which is two fold first is a poorly constructed restorations may be associated with endodontic failure.

\section{References}

[1] Crump MC :- Differential diagnosis in endodontic failure. Dental clinic of North America $1979: 23: 617$

[2] Frank - Inflammatory resorption caused by an adjacent necrotic tooth . J Endodontics 1990 : 16(7) : 339-341

[3] Frank :- Resorption, Perforation and fracture . Dental Clinic North America $1994: 18: 465-487$

[4] Grossman LI :- Endodontic failures . Dental Clinic North America 1972: $16: 79$

[5] Grossman LI :- endodontic practice $\left(10^{\text {th }}\right.$ Edition), Philadelphia 1981, Lea \& Febiger

[6] Ingle JI :- Endodontic success and failure in endodontics . ( $3^{\text {rd }}$ Edition), Philadelhia $1985:$ p 26-53

[7] Oilet S :- Single visit endodontics- A clinical study. J Endodontics 1983 : 9: 147

[8] Richard B Pekrutin :- The incidence of failure following single visit endodontic therapy. J Endodontics 1986 : 12(2) : 68-72

[9] Samuel Seltzer :- Root canal failure in endodontology $2^{\text {nd }}$ Edition, $1988 \mathrm{p} 439-470$

[10] Tronstad 1, Kreshtool D, Barnett F :- Microbial monitoring and result of extra radicular infection. Endo Dental Traumatology, 1990 $: 6: 129-136$.

[11] Ray HA, Trope M :- Periapical status of endodontically treated teeth in relation to the technical quality of root filling and coronal restoration . Int Endodontic J , $1995: 28: 12-18$ 